\title{
Implementation and Different Aspects of Preimplantation Genetic Screening (PGS): A Narrative Review
}

\section{ARTICLE INFO}

Article Type

Review article

\section{Authors}

Zohreh Khezripour $^{1 *}$, M.Sc

Seyedeh Fatemeh Vasegh

Rahimparvar $^{2^{*}}, \mathrm{MD}$

Azam Rahmani², MD

Mohammad Reza Nateghi ${ }^{3}$, iD MD

School of Nursing and Midwifery, Tehran University of Medical Sciences, Tehran, Iran

2 Nursing and Midwifery Care Research Center, School of Nursing and Midwifery, Tehran University of Medical Sciences, Tehran, Iran.

3 Sarem Fertility \& Infertility Research Center (SAFIR) \& Sarem Cell Research Center (SCRC), Sarem Women's Hospital, Iran University of Medical Sciences (IUMS), Tehran, Iran

\section{*Corresponding Author}

Address: School of Nursing and Midwifery, Tehran University of Medical Sciences, Tehran, Iran. Phone: 02130254061

zkhsry@gmail.com

\section{ABSTRACT}

Aneuploidy is considered to be the most common chromosomal disorder and is the most common disorder during IVF treatment. In patients with recurrent abortion that present normal karyotype, preimplantation genetic screening (PGS) has been advised for exploring the presence of aneuploidy which decline abortion rate and result to a higher rate of a healthy pregnancy and live birth. There are various debatable indications for preimplantation genetic screening; the first indication of PGS is maternal age. The genetic material biopsy steps for performing PGS is one of these three techniques; biopsy of the polar body, blastomere in the cleavage stage, and trophectoderm in the blastocyst stage. Three types of protocols are used for controlled ovarian stimulation: the long-term $\mathrm{GnRH}$ agonist, the GnRH antagonist protocol, and the microsimulation protocol. There are various techniques for genetic screening, the most recent one is the "fluorescence in situ hybridization (FISH)" technique for aneuploidy screening; other techniques include "comparative genomic hybridization (aCGH), "single nucleotide polymorphism (SNP)", "quantitative polymerase chain reaction (qPCR)", and "next-generation sequencing technology (NGS)". The aim of this study is to investigate the implementation of PGS and different aspects of this technique to improve pregnancy outcomes. With the possibility of access to assisted reproductive technology and the possibility of oocyte screening and selection of normal oocytes, it is believed that the birth rate of a normal child in couples will increase. Infertility treatment is a costly process and many couples are affected by that. But its beneficial information can help make clinical decisions, and it can be recommended to couples if it improves the outcome of pregnancy, increases the number of live births, and reduces pregnancy loss.

Keywords: Preimplantation Genetic Screening (PGS), Aneuploidy, Pregnancy Outcome, Ovarian Stimulation, Assisted Reproductive Technology (ART). 
روش كار: اين مطالعه مرورى با جستجو در پايكاه اطلاعاتى PubMed،

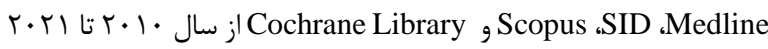

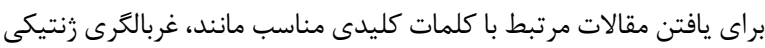

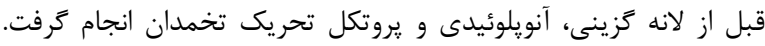

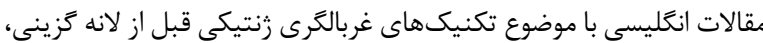

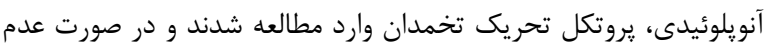

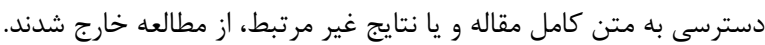

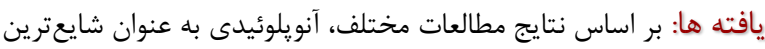

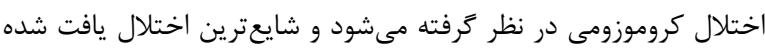

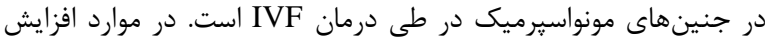

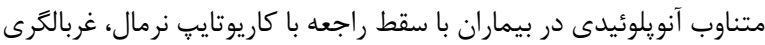

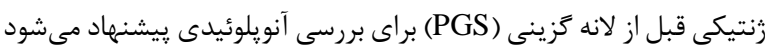

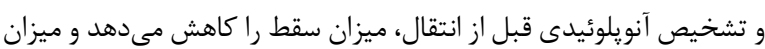

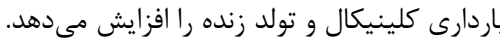

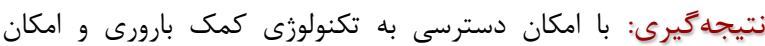

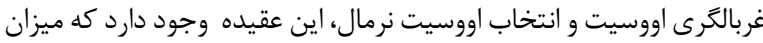

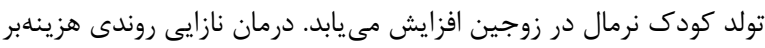

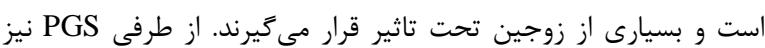

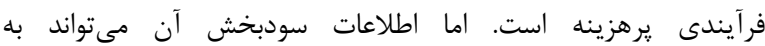

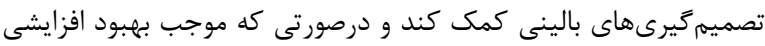

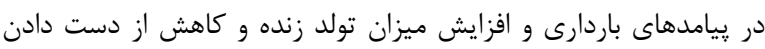

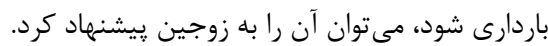

كليد وازمها: غربالكرى زنتيكى قبل از لانه كزينى، آنويلوئيدى، بروتكل تحريك تخمدان، تكنولوزى كمك بارورى

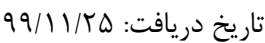

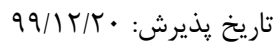

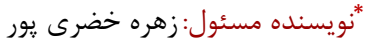

مقدمه

طى لقاح طبيعى در بدن، احتمال رسيدن اسيرم غيرطبيعى به محل لقاح

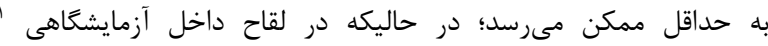

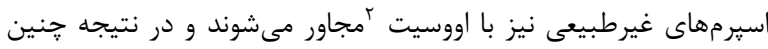

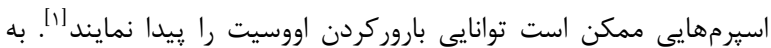

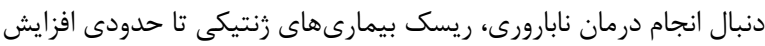

Oocyte

\section{روند اجرا و جنبه و مخاى مختلف

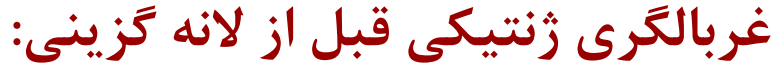 يك مقاله مرورى}

زهره خضرى يور ' "، سيده فاطمه واثق رحيم يرور '، اعظم رحمانى'، (iD) محمدر ضها ناطقى

' دانشكده يرستارى و مامايى، دانشكاه علوم يزشكى تهران، تهران، ايران.

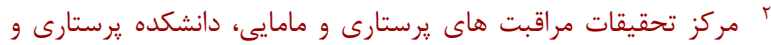

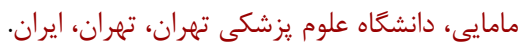

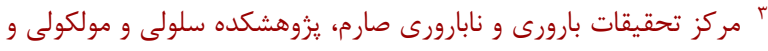

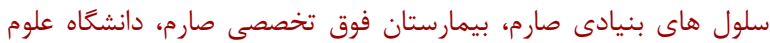
يزشكى ايران، تهران، ايران.

جكيده مقدمه: غربالكَرى زُنتيكى قبل از لانه كَزينى (PGS) مى (Pواند با افزايش

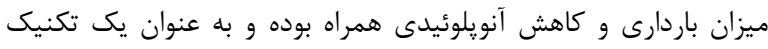

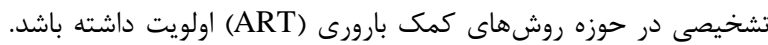

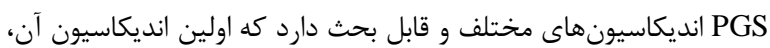

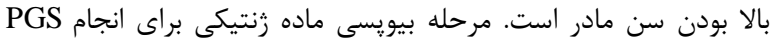

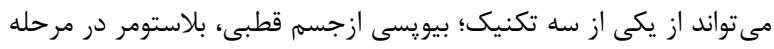

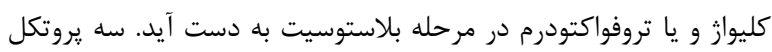

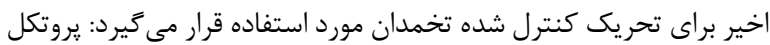

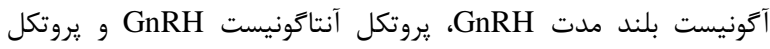

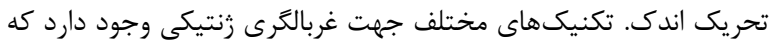

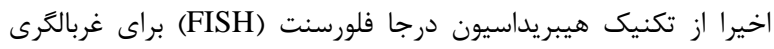

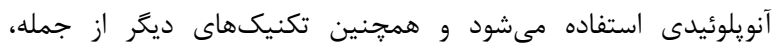

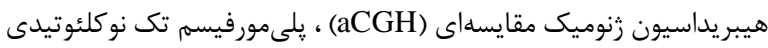

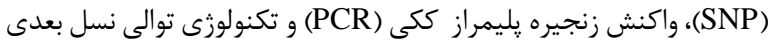

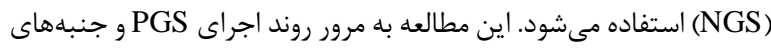

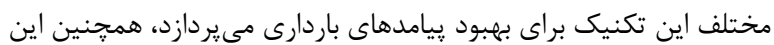

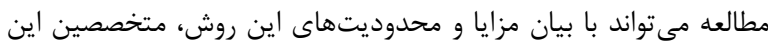

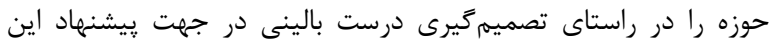

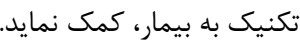

In Vitro Fertilization (IVF)

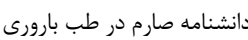

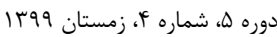




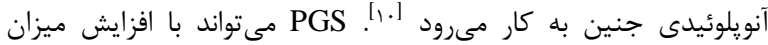

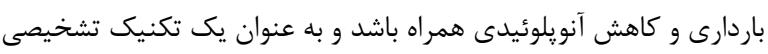

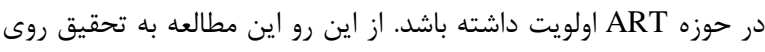

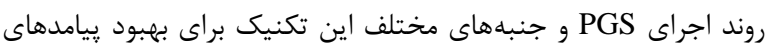

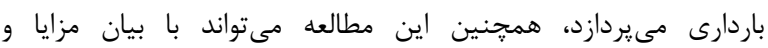

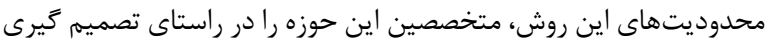

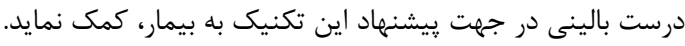

\section{انديكاسيونهاى انجام PGS}

PGS

$$
\text { انديكاسيون هاى آن اشاره مىشود: }
$$

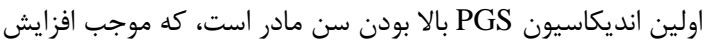

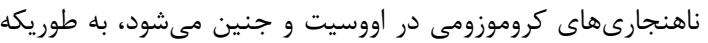

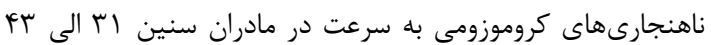

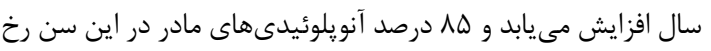

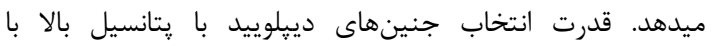
تكنيك PGS، باعث كاهش تعداد جنينهاى انتقالى درهر سيكل،

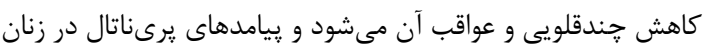
سن بالا، كاهش مى سيابد [1"].

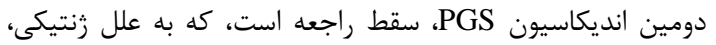

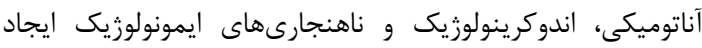

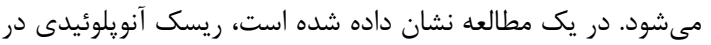

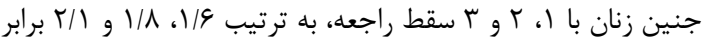

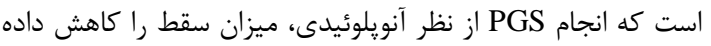
بود [11] سومين انديكاسيون PGS، شكست مكرر تكنيكهاى بارورى است.

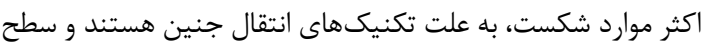

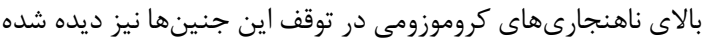

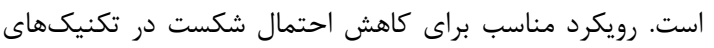

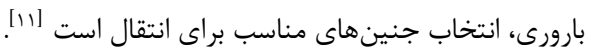

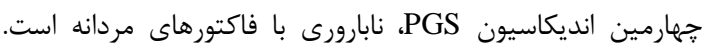

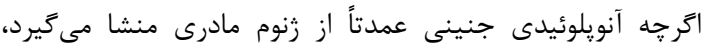

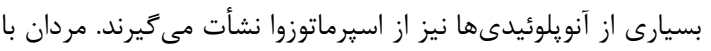
كاريوتيب غيرطبيعى و حذف شدن كروموزم Y اسيرماتوزوا، يك إنى

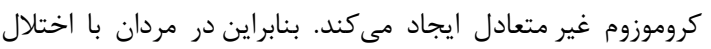

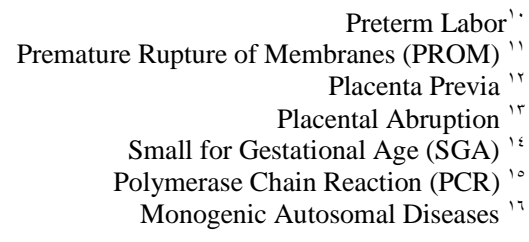

مىيابد و بايد ميزان موفقيت درمانهاى نابارورى نيز افزايش يابد و در

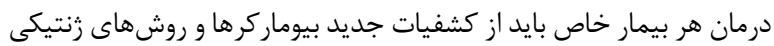

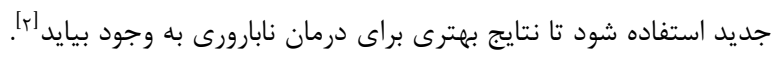

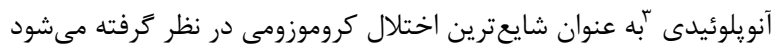

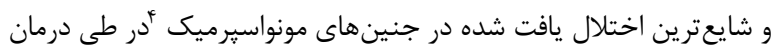

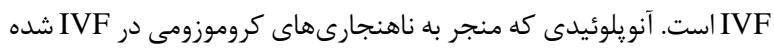

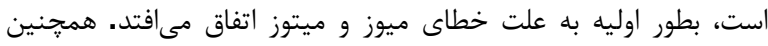

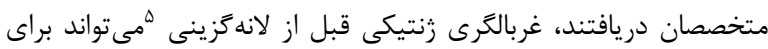

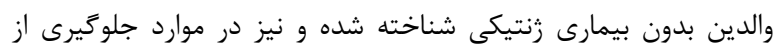

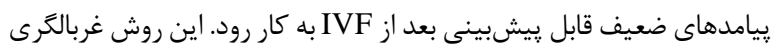

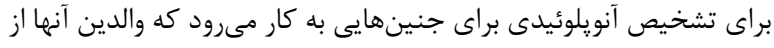

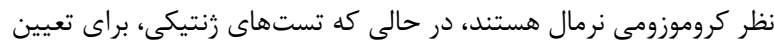

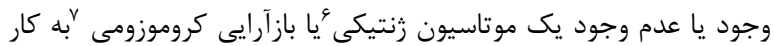

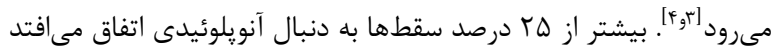

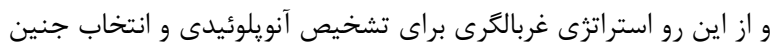

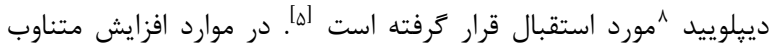

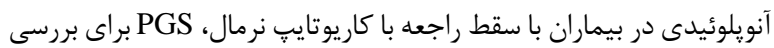

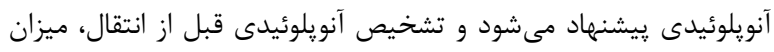

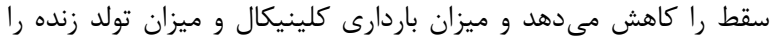

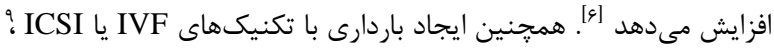

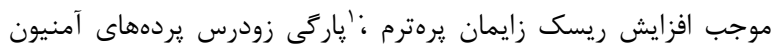

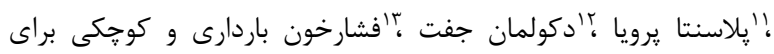

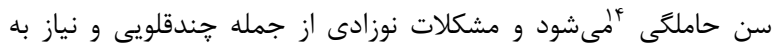

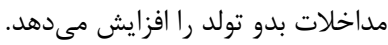

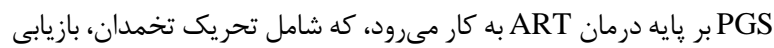

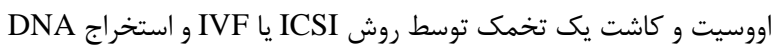

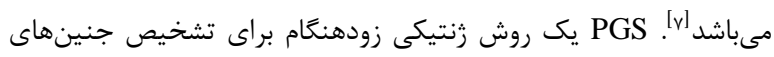

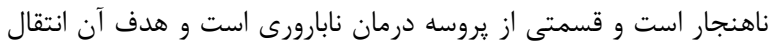

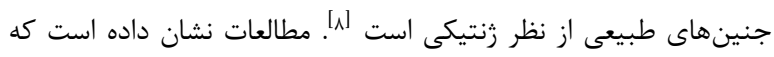
PGS

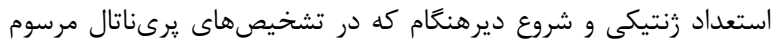

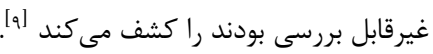

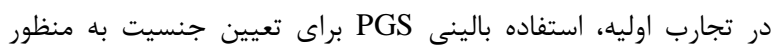

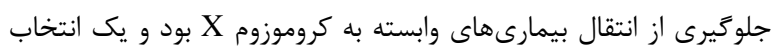

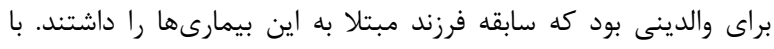

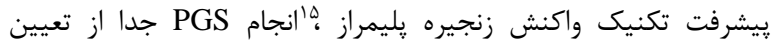

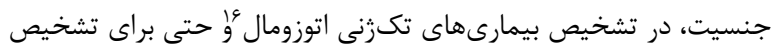

Aneuploidy ${ }^{\circ}$
Monospermic $^{\text {son }}$
Preimplantation Genetic Screening (PGS) $^{\circ}$
Mutation $^{\circ}$
Chromosomal rearrangement
Diploid Embryo $^{\wedge}$
Intracytoplasmic Sperm Injection (ICSI) $^{9}$

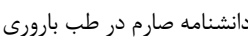

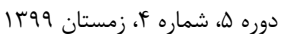




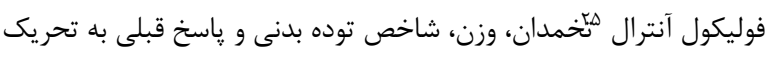

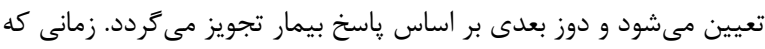

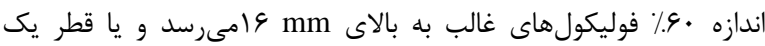

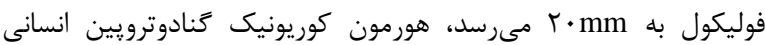

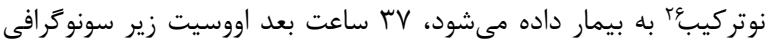

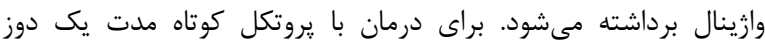

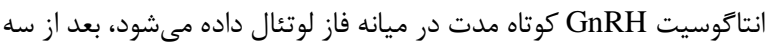

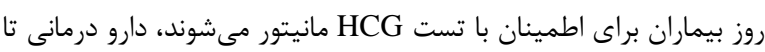

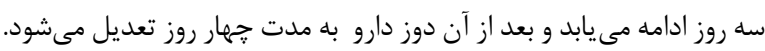

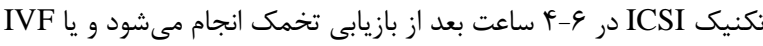

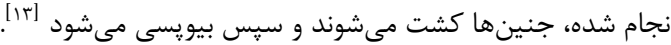

\section{بويسى جنين و تكنيكهاى انجام PGS}

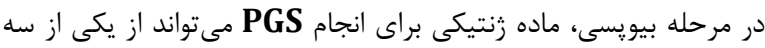

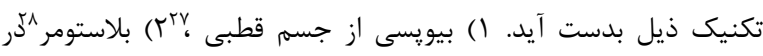

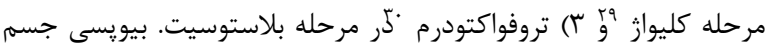

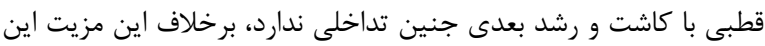

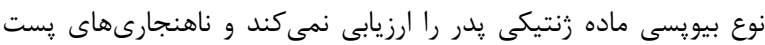

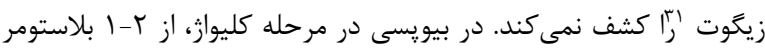

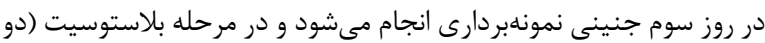

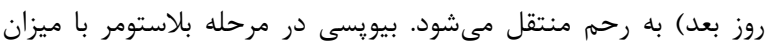

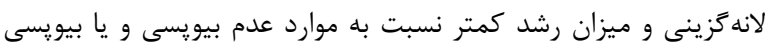

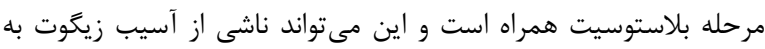

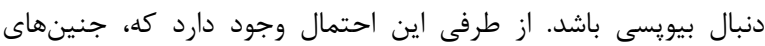

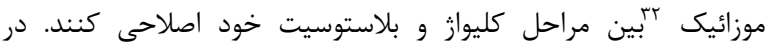

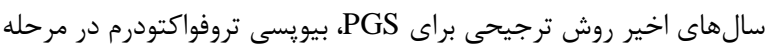

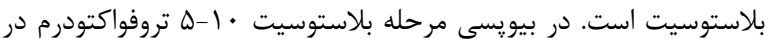

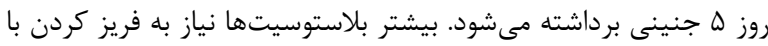

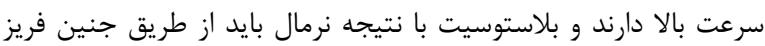

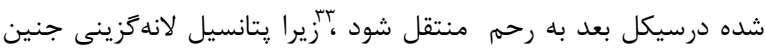

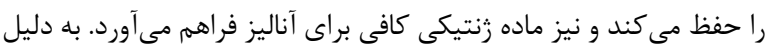

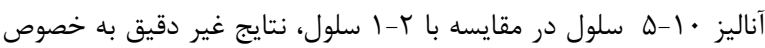

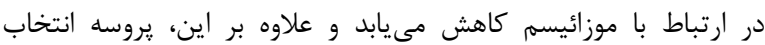

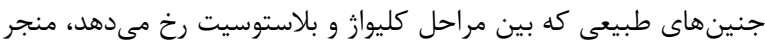

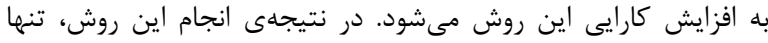

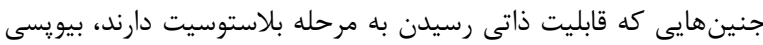

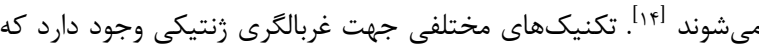

Recombinant Human Chorionic Gonadotropin (r-HCG) ${ }^{r-}$ Polar Body ${ }^{r y}$ Blastomere ${ }^{r /}$ Cleavage Phase ${ }^{r}$ Trophectoderm ${ }^{r}$. Postzygotic

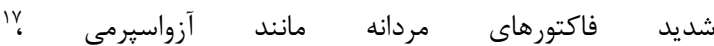

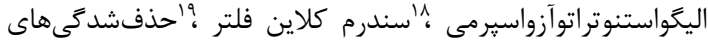
كوجى كروموز Y و حتى مردانى كه آناليز اسيرم آنها مشكل ندارد، PGS ينجمين انديكاسيون PGS استفاده از تخمك اهدايى است. از آنجا

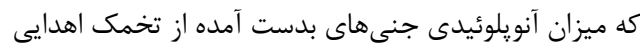

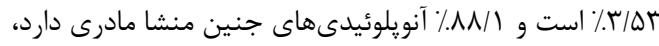
انجام PGS براى اين سيكلها ضرورى به نظر مىرسد [ب، ا11]. ششمين انديكاسيون PGS براى بيمارىهاى مونوزنيك يا زوجين با ريسك بيمارىهاى زنتيك قابل انتقال است [11]. همجنين زوجهايى كه كاريوتيتٍ آنها آنومالى هاى كروموزميى مانند جابجايى هاى متعادل، حذف و موزايسم و كروموزم حلقوى را نشان

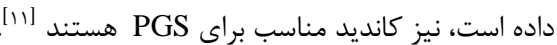

\section{تحريك تخمدان و برداشت اووسيت}

تكنيكهاى كمك بارورى، شامل دستكارى مراحل متعدد لقاح، از تحريك

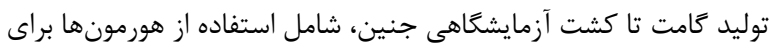

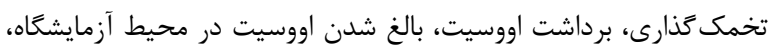

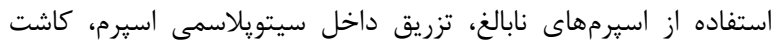

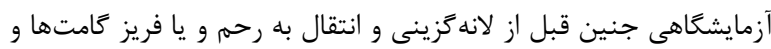

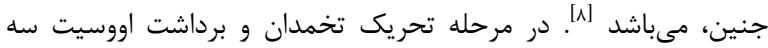
يروتكل اخير براى تحريك كنترل شده تخمدان مورد استفاده قرار مى مئيرد:

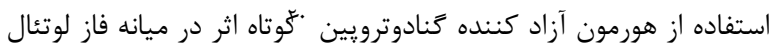

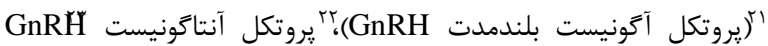

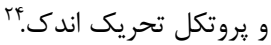

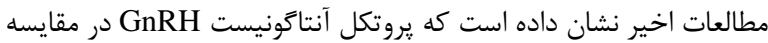

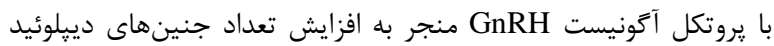

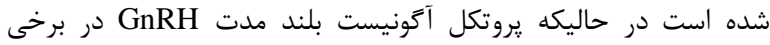

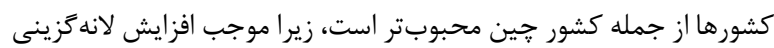

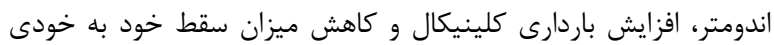

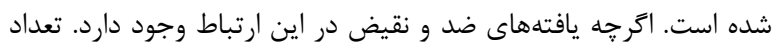

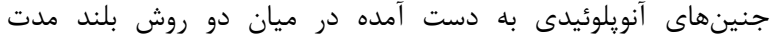

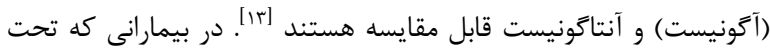
درمان با بروتكل آكونيست قرار مى گَيرند، يكى از داروهاى

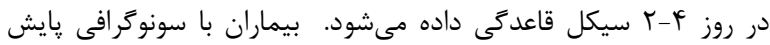

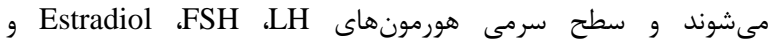
اندازهخيرى مىشود. دوز اوليه حَنادوترويين براساس تعداد

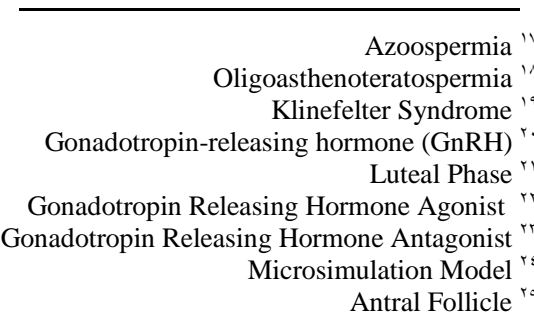

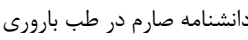




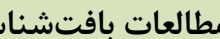

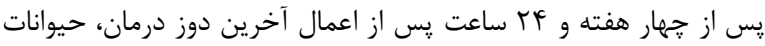

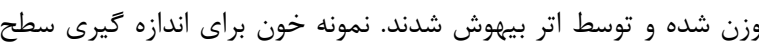

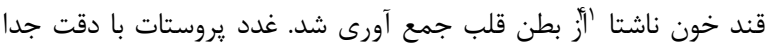

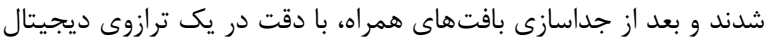

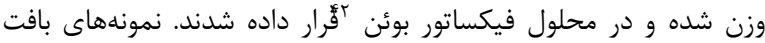

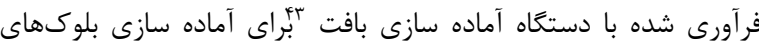

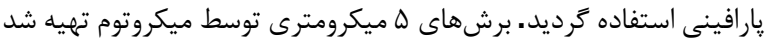

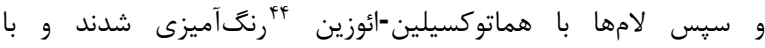
ميكروسكوب نورى تحت بررسى كيفى و كمى قرار كرفتند.

\section{تكنيكهاى ICSI IS IVF}

ميكرواينجكشن (ICSI) تكنيكى است كه با استفاده از روشهاى پيشرفته،

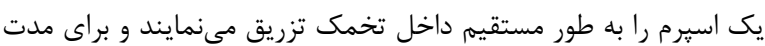

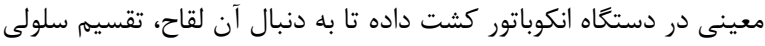

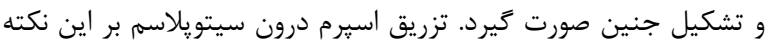

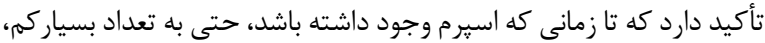

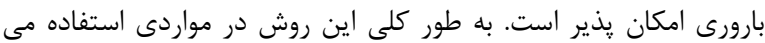
شود كه اسيرم مرد از نظر تعداد، تحرك و يا شكل، كيفيت لازم را نداشته

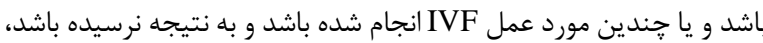

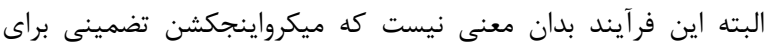

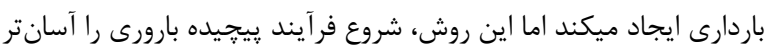

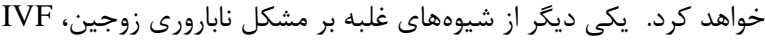

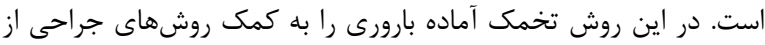

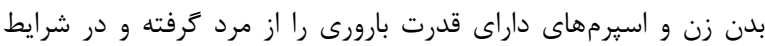

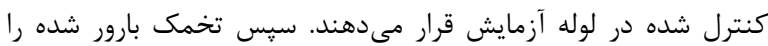

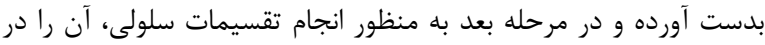

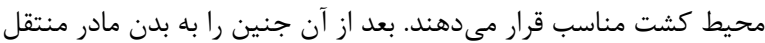

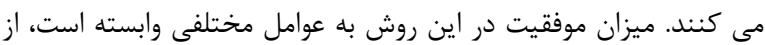

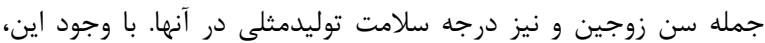
شانس موفقيت از بيمارى به بيمار ديخر متفاوت است و به منظور تخمين

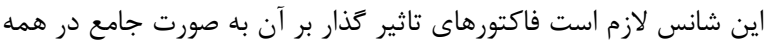

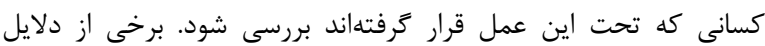

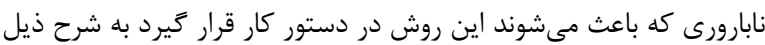

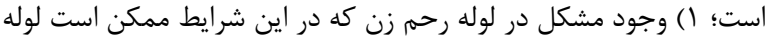

Fasting Blood Sugar (FBS) Bouin Solution (Fixative) ${ }^{\varepsilon r}$ Shandon Eliot Model ${ }^{i r}$ Hematoxylin and Eosin (H\&E) In Vitro Fertilization (IVF) Intracytoplasmic Sperm Injection (ICSI)

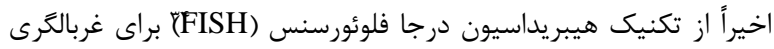

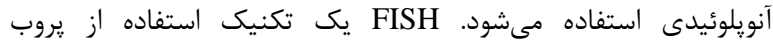

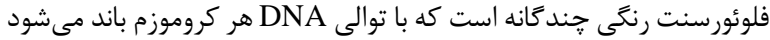

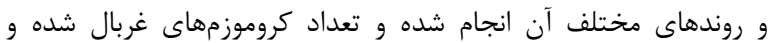

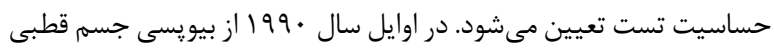

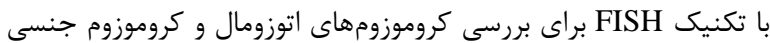

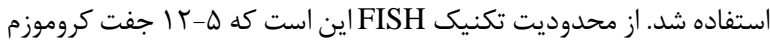

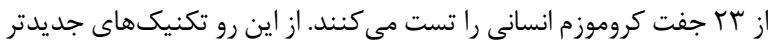

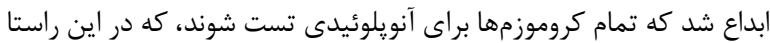

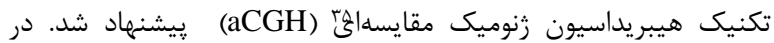

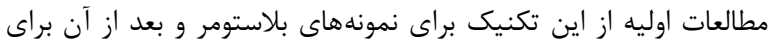

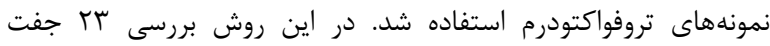

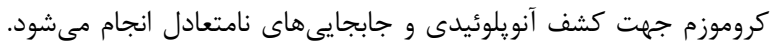

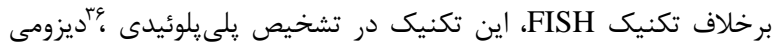

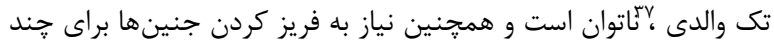

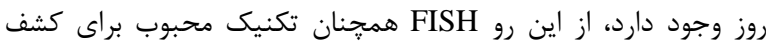

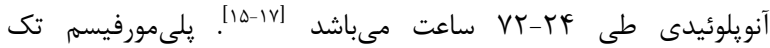

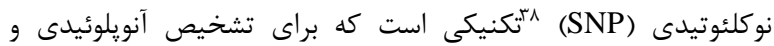

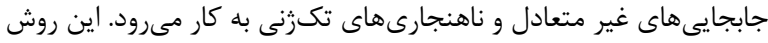
نمىتواند يلى يلوئيدى را تشخيص دهد، اما برخلاف aCGH ديزومى تك آنى

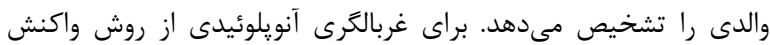

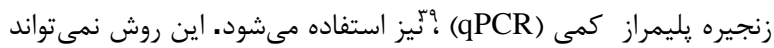
تنها روى يك بلاستومر انجام شود و به نمونه تروفواكتودرم نياز دارد، اما داميا

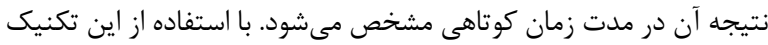

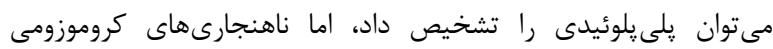
ساختارى و ديزومى تك والدى قابل تشخيص نيستند، از سوى ديخر استفاده راه

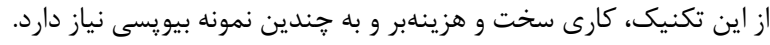

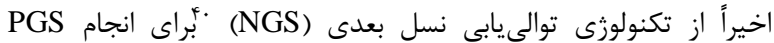

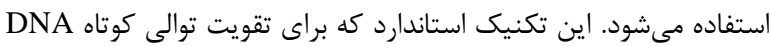

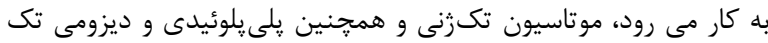

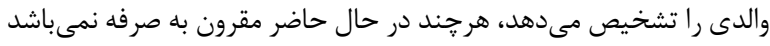

بعد از به كار بردن يكى از اين تكنيكها، يك يا بيشتر از يك جنين حاصل

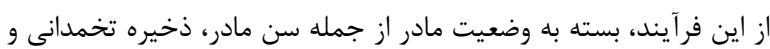

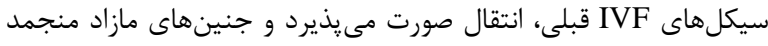
مىشوند تا در صورت نياز در سيكل بعد انتقال يايند [vل

Fluorescence In Situ Hybridization (FISH) Array Comparative Genomic Hybridization (aCGH) Polyploidy ${ }^{r}$ Uniparental Disomy (UPD) Single Nucleotide Polymorphism (SNP) Real-Time Quantitative PCR (qPCR) ${ }^{r}$ Next Generation Sequencing (NGS)

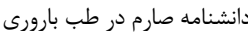

دوره ه، شماره أ، زمستان 


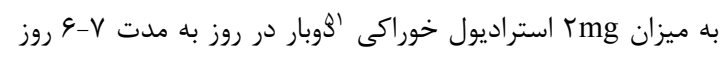

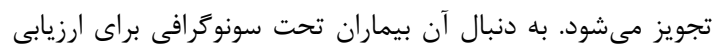

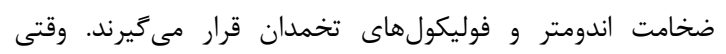

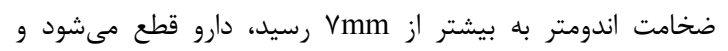

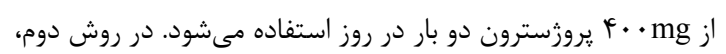

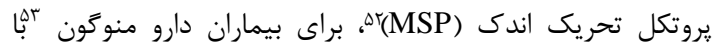

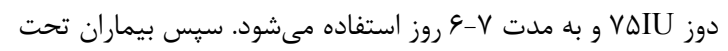

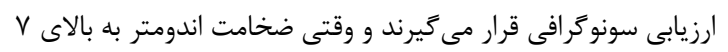

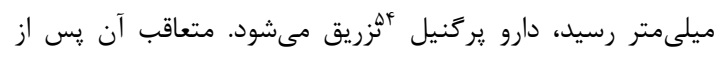

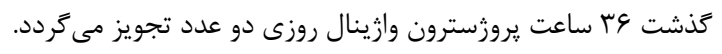

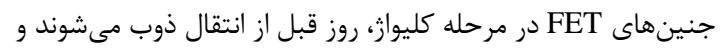

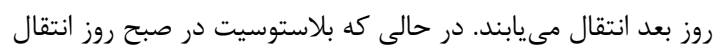
ذوب و همان روز انتقال مى يابد.

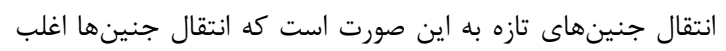

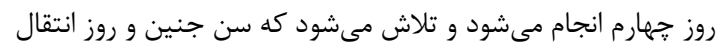

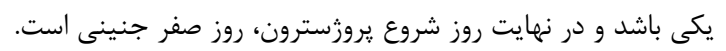

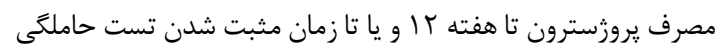

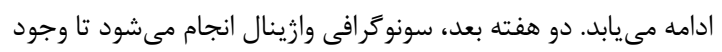
باردارى داخل رحمى تأييد كردد [.r]

\section{نتيجه كَيرى}

با امكان دسترسى به تكنولوزى كمك بارورى و امكان غربالكرى اووسيت و

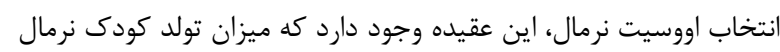

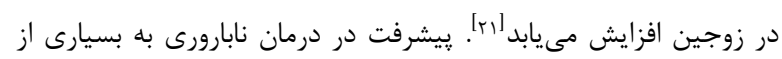

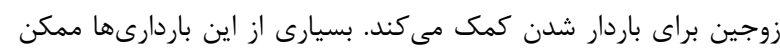

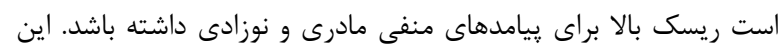

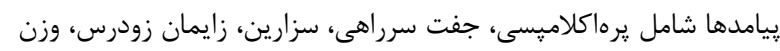

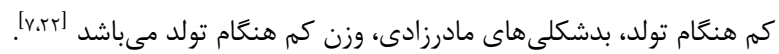

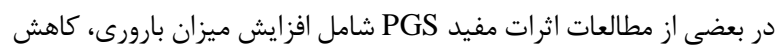

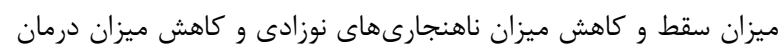

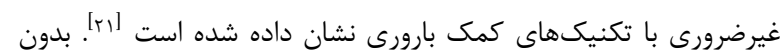

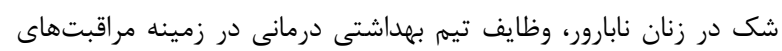

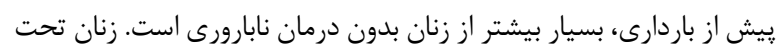

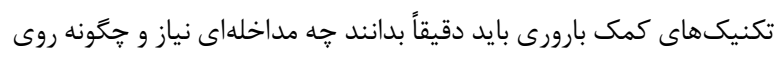

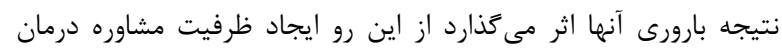

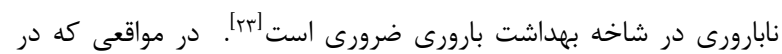
مورد بيماران با انديكاسيون PGS بحث مى شود، متخصص باخد بايستى مزايا و

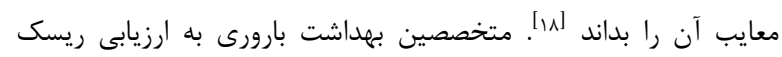

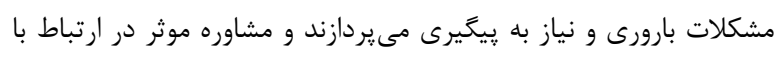

Decapeptyl (Triptorelin) ${ }^{\circ}$ Minimal Stimulation Protocol (MSP) ${ }^{\circ}$ Menogon (Menotropin) or Pregnyl (Human Chorionic Gonadotrophin, HCG) ${ }^{\circ}$
فالوٍ مسدود باشد و يا آسيب ديده باشد. اين امر باعث مىشود كه اسيرم

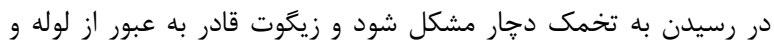

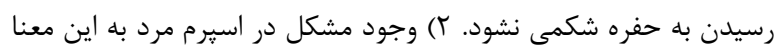

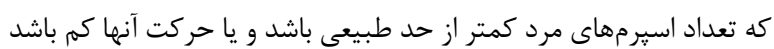

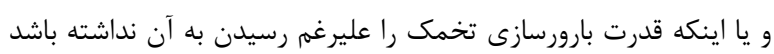

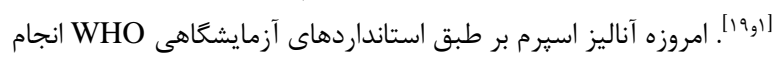

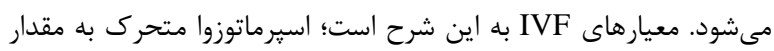

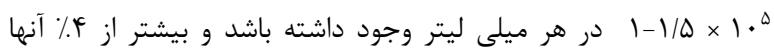

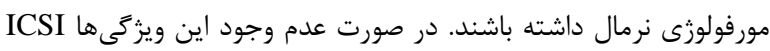

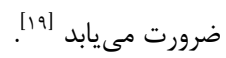

\section{انتقال جنين منجمد و جنين تازه}

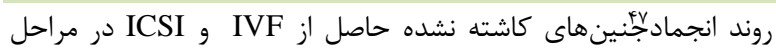

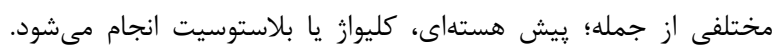

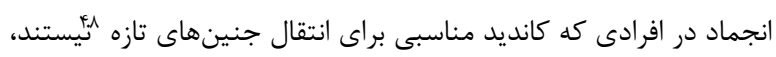

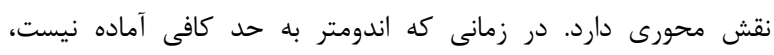

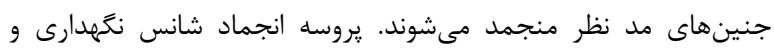

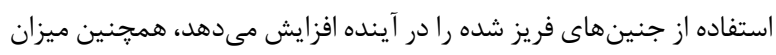

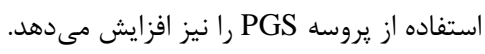

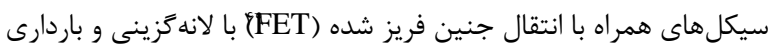
كمترى نسبت به سيكل جنين تازه همراه هستند كه دو علت اساسى دارد:

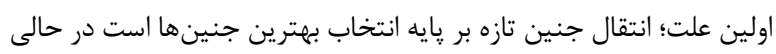

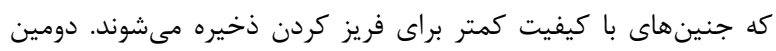

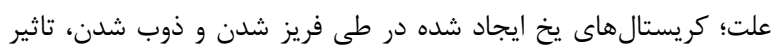

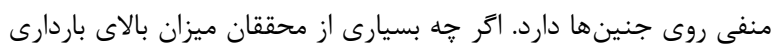

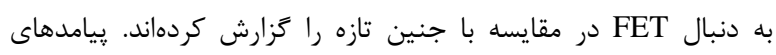

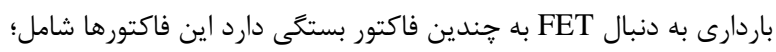

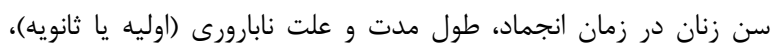

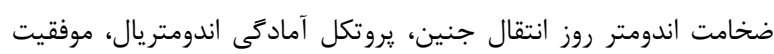

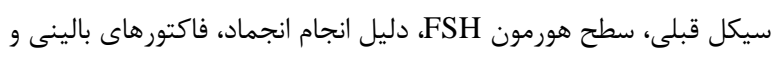

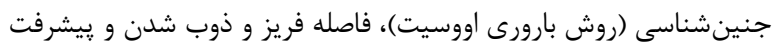

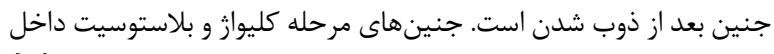

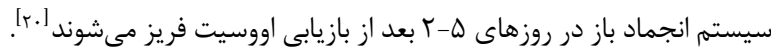

\section{آمادكى اندومتر و انتقال جنين}

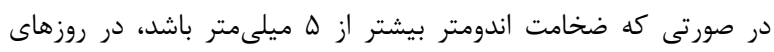

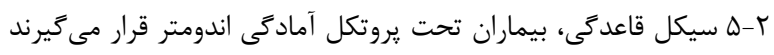
كه به شرح زير است:

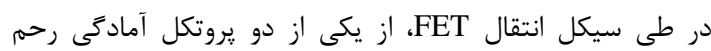

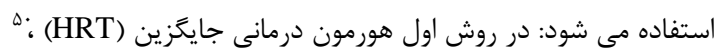


Vitro Fertilization In Ontario: University of Toronto (Canada); 2017.

4. Fesahat F, Montazeri F, Hoseini SM. Preimplantation genetic testing in assisted reproduction technology. Journal of gynecology obstetrics and human reproduction. 2020;49(5):101723.

5. Won SY, Kim H, Lee WS, Kim JW, Shim SH. Preimplantation genetic diagnosis and preimplantation genetic screening: two years experience at a single center. Obstetrics \& gynecology science. 2018;61(1):95-101.

6. Lei C-X, Ye J-F, Sui Y-L, Zhang Y-P, Sun X-X. Retrospective cohort study of preimplantation genetic testing for aneuploidy with comprehensive chromosome screening versus nonpreimplantation genetic testing in normal karyotype, secondary infertility patients with recurrent pregnancy loss. Reproductive and Developmental Medicine. 2019;3(4):205.

7. Feldman B, Orvieto R, Weisel M, Aizer A, Meyer $\mathrm{R}$, Haas J, et al. Obstetric and perinatal outcomes in pregnancies conceived after preimplantation genetic testing for monogenetic diseases. Obstetrics \& Gynecology. 2020.91-782:(4)136;

8. Adamson GD, de Mouzon J, Chambers GM, Zegers-Hochschild F, Mansour R, Ishihara O, et al. International Committee for Monitoring Assisted Reproductive Technology: world report on assisted reproductive technology, 2011. Fertility and sterility. 2018;110(6):106780.

9. Kuliev A, Rechitsky S, Simpson JL. Clinical Outcome of Preimplantation Genetic Testing. Practical Preimplantation Genetic Testing: Springer; 2020. p. 253-73.

10. Sueoka K. Preimplantation genetic diagnosis: an update on current technologies and ethical considerations. Reproductive medicine and biology. 2016;15(2):69-75.

11. Greco E, Litwicka K, Minasi MG, Cursio E, Greco PF, Barillari P. Preimplantation Genetic Testing: Where We Are Today. International Journal of Molecular Sciences. 2020;21(12):4381.

12. Coates A, Bankowski BJ, Kung A, Griffin DK, Munne S. Differences in pregnancy outcomes in donor egg frozen embryo transfer (FET) cycles following preimplantation genetic screening (PGS): a single center retrospective study. Journal of assisted reproduction and genetics. 2017;34(1):71-8.

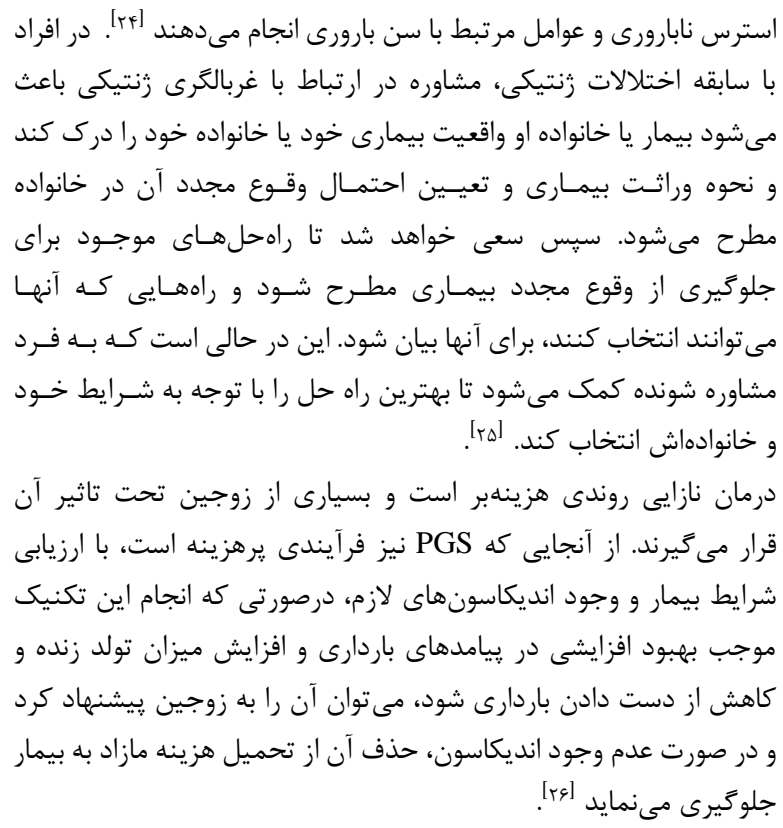

منابع

1. Baktash E, aref. Survey and comparison of gender percentage of boys and girls born following IVF, IUI and ICSI assisted reproductive methods in Al-Zahra Hospital in Tabriz from 2007 to 2016: Tabriz University of Medical Sciences, School of Medicine

2. Szamatowicz M, Szamatowicz J. Proven and unproven methods for diagnosis and treatment of infertility. Advances in medical sciences. 2020;65(1):93-6.

3. Zwingerman RG. A Cost-Effectiveness Analysis of Preimplantation Genetic Screening with In 
attending the University of Benin Teaching Hospital ,Benin City, Nigeria, 2018. Journal of Nursing and Midwifery Sciences. 2019;6(3):125.

24. Foroudi P, Melewar T, Gupta S. Middlesex University Research Repository. Computers in Human Behavior. 2018;80:271e82.

25. Taybi DNGDJADN. Investigating the effect of genetics unit on students' attitudes toward proportion

26. To genetic counseling for abortion therapy. (Ethics Quarterly in Technology, Winter Issue 85).

27. Collins SC, Xu X, Mak W. Cost-effectiveness of preimplantation genetic screening for women older than 37 undergoing in vitro fertilization. Journal of assisted reproduction and genetics. 2017;34(11):1515-22.
13. Li G, Wu Y, Niu W, Xu J, Hu L, Shi H, et al. Analysis of the Number of Euploid Embryos in Preimplantation Genetic Testing Cycles With Early-Follicular Phase Long-Acting Gonadotropin-Releasing Hormone Agonist Long Protocol. Frontiers in Endocrinology. 2020;11:424.

14. Jing S, Luo $\mathrm{K}, \mathrm{He} \mathrm{H}$, Lu C, Zhang S, Tan Y, et al. Obstetric and neonatal outcomes in blastocyststage biopsy with frozen embryo transfer and cleavage-stage biopsy with fresh embryo transfer after preimplantation genetic diagnosis/screening. Fertility and sterility. 2016;106(1):105-12. e4.

15. Zwingerman R. A Cost-Effectiveness Analysis of Preimplantation Genetic Screening with In Vitro Fertilization In Ontario 2017.

16. Parikh FR, Athalye AS, Naik NJ, Naik DJ, Sanap RR, Madon PF. Preimplantation genetic testing: Its evolution, where are we today? Journal of human reproductive sciences. 2018;11(4):306.

17. Totonchi M, Babaabasi B, Najafi $H$, Valojerdi MR, Eftekhari-Yazdi P, Karimian L, et al. Preimplantation Genetic Screening and The Success Rate of In Vitro Fertilization: A ThreeYears Study on Iranian Population. Cell Journal (Yakhteh). 2021;22.(4)

18. Harper JC. Preimplantation genetic screening. Journal of medical screening. 2018;25(1):1-5.

19. Larbuisson A, Raick D, Demelenne S, Delvigne A. ICSI diagnostic: a way to prevent total fertilization failure after 4 unsuccessful IUI. Basic and clinical andrology. 2017;27(1):1-5.

20. Bushaqer NJ, Alkhudhairy NN, Alturaigi ZM, Alhamad RM, Mohawesh WA, Alraka FE, et al. The effect of fresh IVF cycle characteristics on frozen embryo transfer (FET) outcomes. JBRA assisted reproduction. 2020;24(2):135.

21. Schmutzler AG. Theory and practice of preimplantation genetic screening (PGS) . European Journal of Medical Genetics. 2019;62(8):103670.

22. Huang C, Jiang W, Zhu Y, Li H, Lu J, Yan J, et al. Pregnancy outcomes of reciprocal translocation carriers with two or more unfavorable pregnancy histories: before and after preimplantation genetic testing. Journal of Assisted Reproduction and Genetics. 2019;36(11):2325-31.

23. Osian EA, Afemikhe JA, Olorunfemi O, Eweka A. Knowledge and perception of assisted reproductive technology among women 